\title{
The Use of Gingipain DNA Vaccine Against Virulence of Porphyromonas Gingivalis: A Literature Review
}

\author{
Jain $\mathrm{H}^{* 1}$, Kamble $\mathrm{P}^{2}$ and Riyaz Ahmed ${ }^{3}$ \\ ${ }^{1}$ Managing Director, AI Panacea Ltd, Cambridge, England \\ ${ }^{2}$ Former intern, DY Patil Homeopathic College and Hospital, Maharashtra, India \\ ${ }^{3}$ Head, Dental Department, Lala Lajpat Rai Memorial Medical College, Meerut, India
}

*Corresponding author: Jain H, Managing Director, AI Panacea Ltd, Cambridge, England, Tel: +447448871521, E-mail: drhansajain25@gmail.com

Citation: Jain H, Kamble P, Riyaz Ahmed (2018) The Use of Gingipain DNA Vaccine against Virulence of Porphyromonas Gingivalis: A Literature Review. J Cell Biol Histol 1(1): 106. doi: 10.15744/2638-082X.1.106

Received Date: April 16,2018 Accepted Date: May 28, 2018 Published Date: June 12, 2018

\begin{abstract}
Background: Porphyromonas gingivalis (P. gingivalis) is a primary etiologic agent of generalized aggressive and chronic forms of periodontitis. It releases toxins called 'gingipains'.

Highlights: Gingipains, (Arginine-and Lysine-specific cysteine proteinases) are proteases that are critical virulence factors in the progression of periodontitis and degradation of cytokines expressed during inflammation. HRgpA and RgpB (products of two distinct but related genes) induce vascular permeability by activation of the kallikrein/kinin pathway and activating the blood coagulation system which in turn induces gingival crevicular fluid production and progression of inflammation leading to alveolar bone loss.
\end{abstract}

Conclusion: The review focuses on various DNA vaccines which can induce protection against virulence of gingipains.

Keywords: Porphyromonas Gingivalis; Chronic Periodontitis; hgp44; Gingipains

\section{Introduction}

Periodontium surrounds the root part of teeth and is composed of gingiva, periodontal ligament, cementum and alveolar bone. Periodontitis is an infectious disease afflicting the tissues anchoring the teeth, eventually leading to loss of teeth [1,2]. Data evaluated by the NHANES 2009-10 estimated that over 47\% of the US population, representing 64.7 million adults has periodontitis [3]. The literature suggests that periodontitis is linked with a mosaic of numerous microbiota of the oral biofilm. This biofilm induces local as well as systemic inflammatory responses, thus causing periodontal degradation. It has been decades since the recognition of the oral microbial diversity in humans, and today over 800 species have been identified [4,5].

Studies have reported that only 10-20 bacterial pathogens present in the dental plaque called the periodontopathic microbiota, lead to causation of destructive periodontitis. These include Porphyromonas gingivalis ( $P$. gingivalis), Treponema denticola (T. denticola), Tannerella forsythia (T. forsythia) and Aggregatibacter actinomycetemcomitans (Aa) [6-8]. Socransky et al. stated that periodontopathogens can be segregated on the color-coded system reflecting cluster analysis, community ordination and associated disease severity. Amongst these groups, 'red complex' including three species i.e. P. gingivalis, T. forsythia and T. denticola are considered the most pivotal in causing periodontitis. Among the red complex, $P$. gingivalis is the most effortless to grow and genetically manipulative, so it became the most investigated and examined periodontal bacterium $[9,10]$.

\section{Porphyromonas gingivalis, Gingipain, and Periodontitis}

P. gingivalis, under the environmental conditions that prevail during the host inflammatory response, is able to out-compete other bacteria present in the subgingival microflora and reach important levels within the community [2]. It is a black-pigmented gramnegative anaerobic periodontopathogen. A varied number of virulence factors have been recognized behind the pathogenicity of $P$. gingivalis, namely fimbriae, lipopolysaccharides, various proteases and hemagglutinins [2]. Ogawa and Hamada were the first to report the hemagglutinating activity and erythrocyte agglutinating activity of P. gingivalis. i.e. 381 FimA fimbria and their oligopeptide segments [11].

Gingipains are highly virulent cysteine proteinases secreted by $P$. gingivalis causing progression of periodontitis. Among other functions, they degrade inflammatory cytokines thereby down-regulating the host response and reducing inflammation. Gingipains 
are also involved in adherence and colonization of epithelial cells, vascular permeability, haemagglutination and hemolysis of erythrocytes by activating kallikrein/kinin pathway and the blood coagulation system, and it also plays an important role in degradation of host proteins and tissues $[12,13]$.

Peptidases are a pivotal part in the causation of infection caused by $P$. gingivalis. Most of the ones listed are cysteine peptidases including lysine-specific cysteine protease i.e. lys gingipains (Kgp) and arginine-specific cysteine protease i.e. arg-gingipains A and B (HRgpA and RgpB). These two are the major virulence factors of this pathogen. Gingipains are cell-surface-anchored or soluble peptidases which represent around $85 \%$ of the total extracellular proteolytic activity of $P$. gingivalis [14-16]. There are two genes called $\operatorname{rgpA}$ and $\operatorname{rgpB}$ encoding HRgpA and RgpB whereas only one gene kgp encodes lysine-specific gingipain $\mathrm{K}$ (Kgp) [11-17]. $r g p A$ and $\operatorname{rgp} B$ are similar in structure, except for the fact that $\operatorname{rgp} B$ does not contain the C-terminal adhesin domains of $\operatorname{rgp} A$. There are three main components of $r g p A$ and $\mathrm{kgp}$ which include a propeptide domain, a catalytic domain, and a C-terminal adhesion domain [11,18]. The adhesion domain contains four subdomains enlisting Hgp (15, 17, 27 and 44) [16]. hagA and tla encode the parts of the hemagglutinin domains of the $k g p$ and $r g p A$ genes [17]. It is also observed that the adhesion subdomains are highly homologous between $\operatorname{rgp} A$ and $k g p$. In comparison to $\operatorname{rgp} A, \operatorname{rgp} B$ consists of a propeptide domain and a catalytic domain which does not contain an adhesion domain [19].

The literature suggests that the virulence of $P$. gingivalis decreases in Rgp- and/or Kgp-deficient mutants [19]. Rgp is responsible for producing surface proteins on $P$. gingivalis enlisting fimbrilin a $75 \mathrm{kDa}$ protein, and prolyl tripeptidyl peptidase [18]. HRgpA and $\operatorname{RgpB}$ cause generation of bradykinin by activating prekallikrein, whereas Kgp generates kinin directly from its precursor [20]. Gingipains are even pivotal in maintaining a sustainable environment for bacteria to proliferate in the periodontal pocket [15].

\section{DNA Vaccines and Periodontitis}

A number of techniques are being applied for development of vaccines to prevent periodontitis; in 1992 Evans et al. concluded that fimbriae from P. gingivalis are cardinal virulent factors which can be used as a target antigen and an effective vaccine against periodontitis [21]. Use of the whole cell has also been quoted; in this, the entire cell was inoculated into the host to bring about immunization [22]. In 2011 Kudyar stated use of outer membrane and observed that its use decreased activities of collagenase, gelatinase, and cysteine protease in gingival tissue [22]. Another study also proposed the use of outer membrane vesicles of $P$. gingivalis as a mucosal immunogen and adjuvant for a periodontitis vaccine. It was observed that there was inhibition of gingipain proteolytic activity along with a decrease in viability of $P$. gingivalis [23]. Wilensky et al tested recombinant and native HRgpA vaccination against $P$. gingivalis infection. Following immunization, the immune response was shifted towards anti-inflammatory response and there was a reduction in bone loss [24].

DNA vaccines have emerged as a favorable alternative to outmoded protein-based vaccines inducing immune responses. They are safe and stable to use, are specific in the induction of immune response to the antigen of interest including both B and T-cell responses, easy to manufacture and targets a wide variety of infectious diseases. The disadvantages include poor immunogenicity in clinical trials as naked plasmid DNA is often weak and is subjected to degradation by nucleases and demonstrate inefficient delivery to immune cells $[25,26]$. In a study by Yonezawa et al., mice were immunized with $\operatorname{rgp} A$ DNA vaccine, controls were non-immunized mice and mice immunized with control plasmid pVAX1 (2.5 lg/mice) by Gene Gun. It was observed that there was a change in cytokine levels. The production of interleukin (IL)-2, 4, 5 and 12 was found to be elevated, whereas, interferon (IFN) $-\gamma$ was lower in the serum of the DNA vaccine-immunized mice than in the serum of the control mice. This lowering of IFN- $\gamma$ levels plays a major role in the protection of mice against bacterial attack. It was concluded that the $\operatorname{rgp} A$ DNA vaccine may induce humoral and cellular immune responses to bring about protection against $P$. gingivalis challenge [27]. In 1998 Genco et al. carried out a study to evaluate the effect of immunization with gingipains on the ability of $P$. gingivalis of colonization and invasion in mice. Mice were immunized with the $95-\mathrm{kDa}$ gingipain $\mathrm{R} 1$, the $50-\mathrm{kDa}$ gingipain $\mathrm{R} 2$, or multiple antigenic peptides (MAP)conjugated gingipain R-derived peptides and then were subjected to challenge with $P$. gingivalis. It was observed that antibodies directed towards the amino-terminal region of the catalytic domain of gingipain $\mathrm{R}$ had capability to induce a protective immune response against $P$. gingivalis infection [28].

In another study by Kuboniwa et al. the role of the catalytic domain of Kgp (Kgpcd) in hemoglobin binding by P. gingivalis was evaluated. To enhance antibody responses, a signal sequences was engineered into the plasmid vector which provided antigen secretion. The constructed DNA vaccines were even used in a genetic immunization strategy against $P$. gingivalis virulence to examine the effectiveness of Kgpcd as an immunogen. It was observed that generated antibodies were able to inhibit Lys- and Arg- specific proteolytic activities. The study showed that supplemented hemoglobin was the only iron source for growth in the iron-restricted condition. The Kgpcd acted as a hemoglobin-binding protein of $P$. gingivalis. It can be summarized that Kgpcd acts as a possible immunogen to induce a protective response to $P$. gingivalis infection [29].

Yonezawa et al. in 2001 demonstrated that in order to induce a protective immune response against the periodontopathogen $P$. gingivalis, authors manufactured an $r g p A$ DNA vaccine. In the process, they immunized mice intradermally with DNA carrying $r g p A$ using Gene Gun. The vaccine was able to induce high levels of serum antibodies against $P$. gingivalis. It was also observed that sera from the immunized mice had an almost negligible proteolytic activity of Rgp A \& B and binding action of $P$. gingivalis 
to a type I collagen was also reduced to a minimum. Moreover, sera were effective in decreasing hemagglutination induced by $P$. gingivalis. The results suggested that the vaccine was capable of reducing the virulence of $P$. gingivalis [30].

Miyachi et al. used $\operatorname{rgpA}$ DNA vaccine to immunize mice using a Gene Gun or intranasal route. The immunization was followed by injecting a specified quantity of $P$. gingivalis. Immunization evoked IgG and sIgA responses against $P$. gingivalis. There was a decreased loss of alveolar bone in both the groups but the effect was more prominent in the intranasal immunization group than in the Gene Gun group. Thus, the results suggested that immunization with the $\operatorname{rgp} A$ DNA vaccine through the nasal cavity is a competent method for the prevention of alveolar bone loss incited by infection with P. gingivalis [31].

In 2011, Muramatsu et al. also evaluated the protective effect of HRgpA DNA vaccine against virulence of gingipains on bone loss. They divided 6 weeks old female BALB/c mice into 8 groups including a non-immunized group and groups receiving vaccines containing the $\mathrm{rgpA}$ catalytic domain [pcat], the HGP44 domain-coding region (phgp44), the HGP 15-27 domain-coding region (phgp15-27), or the N-terminal (phgp44H] or C-terminal (phgp44T) half of the HGP44 domain-coding region against infection by $P$. gingivalis. There was a significant elevation of specific IgGs against $P$. gingivalis. This was highest in the $r g p A D N A$ vaccine and in those immunized with phgp44. The reduction in loss of bone was observed to be most in $\operatorname{rgp} A$ DNA group followed by phgp44 group [32]. The advantages that DNA vaccines include are that they are simple, easy to manufacture and stable by nature [22]. Stimulation of adaptive and innate immunity reduction of lethality protects bacterial binding to hemoglobin; vaccine protein binds to hemoglobin and induces protective response and induce specific antibodies against enzymes avoidance of safety issues, unlike live vaccines, they do not revert back into virulent forms are other benefits [29,30,33]. Along with this, there are few problems also related to DNA vaccine, such as limited potency in the clinical setting $[34,35]$.

\section{Conclusion}

Thus, it can be concluded that DNA vaccines are definitely effective in reducing the virulence of $P$. gingivalis. It can be used as an effective vaccine to prevent or reduce pathogenicity during the periodontal inflammation.

\section{Acknowledgement}

The authors would like to thank Mrs. Shanti Jha and Mrs. Parul Jain. This was a self-funded research.

\section{References}

1. Zheng Y, Hou J, Peng L, Zhang X, Jia L, et al. (2014) The pro-apoptotic and pro-inflammatory effects of calprotectin on human periodontal ligament cells. PLoS One 9: e110421.

2. Percival RS, Marsh PD, Devine DA, Rangarajan M, Aduse-Opoku J, et al. (1999) Effect of temperature on growth, hemagglutination, and protease activity of Porphyromonas gingivalis. Infect Immun 67: 1917-21.

3. Papapanou PN (2012) The prevalence of periodontitis in the US: forget what you were told. J Dent Res 91: 907-8.

4. Souto R, Silva-Boghossian CM, Colombo AP (2014) Prevalence of Pseudomonas aeruginosa and Acinetobacter spp. in subgingival biofilm and saliva of subjects with chronic periodontal infection. Braz J Microbiol 45: 495-501.

5. Siqueira JF Jr, Rocas IN (2010) The oral microbiota: general overview, taxonomy, and nucleic acid techniques. Methods Mol Biol 666: 55-69.

6. Haffajee AD, Socransky SS (2000) Microbiology of periodontal diseases: introduction. Periodontol 38: 9-12.

7. Socransky SS, Haffajee AD (2000) Periodontal microbial ecology. Periodontol 38: 135-87.

8. Li N, Collyer CA (2011) Gingipains from Porphyromonas gingivalis - Complex domain structures confer diverse functions. Eur J Microbiol Immunol (Bp) 1: 41-58.

9. Hajishengallis G, Lamont RJ (2012) Beyond the red complex and into more complexity: the polymicrobial synergy and dysbiosis (PSD] model of periodontal disease etiology. Mol Oral Microbiol 27: 409-19.

10. Ximenez-Fyvie LA, Haffajee AD, Socransky SS (2000) Comparison of the microbiota of supra and subgingival plaque in health and periodontitis. J Clin Periodontol 27: 648-57.

11. Ogawa T, Hamada S (1994) Hemagglutinating and chemotactic properties of synthetic peptide segments of fimbrial proteinfrom Porphyromonas gingivalis. Infect Immun 62: 3305-10

12. Abdi K, Chen T, Klein BA, Tai AK, Coursen J, et al. (2017) Mechanisms by which Porphyromonas gingivalis evades innate immunity. PLoS One 12: e0182164.

13. Li N, Collyer CA. Gingipains from Porphyromonas gingivalis - Complex domain structures confer diverse functions. Eur J Microbiol Immunol (Bp) 1: 41-58.

14. de Diego I, Veillard F, Sztukowska MN, Guevara T, Potempa B, et al. (2014) Structure and mechanism of cysteine peptidase gingipain K (Kgp), a major virulence factor of Porphyromonas gingivalis in periodontitis. J Biol Chem 289: 32291-302.

15. Takii R, Kadowaki T, Baba A, Tsukuba T, Yamamoto K (2005) A functional virulence complex composed of gingipains, adhesins, and lipopolysaccharide shows high affinity to host cells and matrix proteins and escapes recognition by host immune systems. Infect Immun 73: 83-93.

16. O'Brien-Simpson NM, Paolini RA, Hoffmann B, Slakeski N, Dashper SG, et al. (2001) Role of RgpA, RgpB, and Kgp proteinases in virulence of Porphyromonas gingivalis W50 in a murine lesion model. Infect Immun 69: 7527-34.

17. Simpson W, Olczak T, Genco CA (2004) Lysine-specific gingipain K and heme/hemoglobin receptor HmuR are involved in heme utilization in Porphyromonas gingivalis. Acta Biochim Pol 51: 253-62.

18. Galimanas V, Hall MW, Singh N, Lynch MD, Goldberg M, et al. (2014) Bacterial community composition of chronic periodontitis and novel oral sampling sites for detecting disease indicators. Microbiome 2: 32 .

19. Kadowaki T, Nakayama K, Okamoto K, Abe N, Baba A, et al. (2000) Porphyromonas gingivalis proteinases as virulence determinants in progression of periodontal diseases. The Journal of Biochemistry 128: 153-9. 
20. Rapala-Kozik M, Bras G, Chruscicka B, Karkowska-Kuleta J, Sroka A, et al. (2011) Adsorption of components of the plasma kinin-forming system on the surface of Porphyromonas gingivalis involves gingipains as the major docking platforms. Infect Immun 79: $797-805$.

21. Evans RT, Klausen B, Sojar HT, Bedi GS, Sfintescu C, et al. (1992) Immunization with Porphyromonas (Bacteroides) gingivalis fimbriae protects against periodontal destruction. Infect Immun 60: 2926-35.

22. Kudyar N, Dani N, Mahale S (2011) Periodontal vaccine: A dream or reality. J Indian Soc Periodontol 5: 115-20.

23. Nakao R, Hasegawa H, Dongying B, Ohnishi M, Senpuku H (2016) Assessment of outer membrane vesicles of periodontopathic bacterium Porphyromonas gingivalis as possible mucosal immunogen. Vaccine 34: 4626-34.

24. Wilensky A, Potempa J, Houri-Haddad Y, Shapira L (2017) Vaccination with recombinant RgpA peptide protects against Porphyromonas gingivalis-induced bone loss. J Periodontal Res 52: 285-91.

25. Jafari N, Abediankenari S (2015) Phage particles as vaccine delivery vehicles: concepts, applications and prospects. Asian Pac J Cancer Prev 16: 8019-29.

26. Farris E, Brown DM, Ramer-Tait AE, Pannier AK (2016) Micro- and nanoparticulates for DNA vaccine delivery. Exp Biol Med (Maywood) 241 : $919-29$.

27. Yonezawa H, Kato T, Kuramitsu HK, Okuda K, Ishihara K (2005) Immunization by Arg-gingipain A DNA vaccine protects mice against an invasive Porphyromonas gingivalis infection through regulation of interferon-gamma production. Oral Microbiol Immunol 20: 259-66.

28. Genco CA, Odusanya BM, Potempa J, Mikolajczyk-Pawlinska J, Travis J (1998) A peptide domain on gingipain R which confers immunity against Porphyromonas gingivalis infection in mice. Infect Immun 66: 4108-14.

29. Kuboniwa M, Amano A, Shizukuishi S, Nakagawa I, Hamada S (2001) Specific antibodies to Porphyromonas gingivalis Lys-gingipain by DNA vaccination inhibit bacterial binding to hemoglobin and protect mice from infection. Infect Immun 69: 2972-9.

30. Yonezawa H, Ishihara K, Okuda K (2001) Arg-gingipain a DNA vaccine induces protective immunity against infection by Porphyromonas gingivalis in a murine model. Infect Immun. 69: 2858-64.

31. Miyachi K, Ishihara K, Kimizuka R, Okuda K. Arg-gingipain A DNA vaccine prevents alveolar bone loss in mice. J Dent Res 86: 446-50.

32. Muramatsu K, Kokubu E, Shibahara T, Okuda K, Ishihara K (2011) HGP44 induces protection against Porphyromonas gingivalis-Induced alveolar bone loss in mice. Clin Vaccine Immunol 18: 888-91.

33. Yonezawa H, Kato T, Kuramitsu HK, Okuda K, Ishihara K (2005) Immunization by Arg-gingipain A DNA vaccine protects mice against an invasive Porphyromonas gingivalis infection through regulation of interferon- $\gamma$ production Oral Microbiol Immunol 20: 259-66

34. Gordy JT, Luo K, Zhang H, Biragyn A, Markham RB (2016) Fusion of the dendritic cell-targeting chemokine MIP3a to melanoma antigen Gp100 in a therapeutic DNA vaccine significantly enhances immunogenicity and survival in a mouse melanoma model. J Immunother Cancer 4: 96.

35. Kutzler MA, Weiner DB (2008) DNA vaccines: ready for prime time? Nat Rev Genet 9: 776-88.

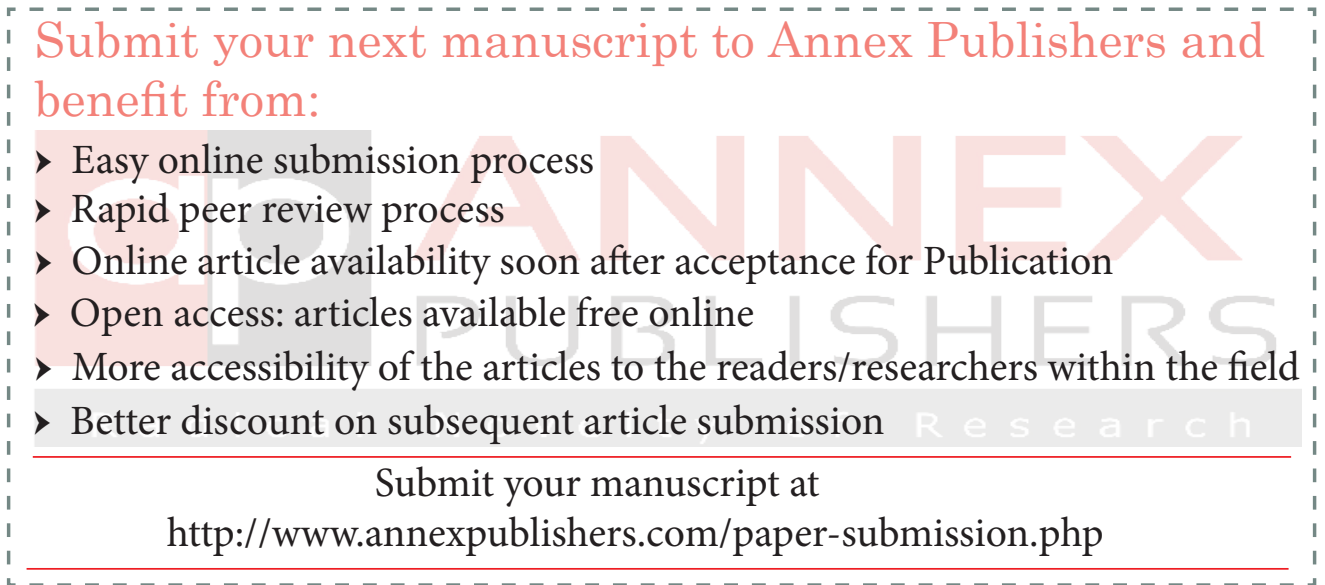

